\title{
EL FEDERALISMO ARGENTINO
}

\section{Emilia Raquel Lerner (*)}

La República Argentina tiene una organización federal de gobierno, que se expresa en la existencia de 23 provincias.

Las Provincias representan en Argentina una proporción importante y creciente del sector público global. La ampliación de las funciones de los estados provinciales se verifica fundamentalmente a partir de la década del 70. En el período anterior, y fundamentalmente a partir de mediados de la década del 40, el Estado expande sus funciones a través de políticas ejecutadas fundamentalmente de forma centralizada.

En la década del 70 se produce la transferencia hacia las provincias de la educación primaria, servicios de atención médica, y de otros servicios sociales como vivienda y saneamiento urbano.

Durante el presente ejercicio se produjo la transferencia de escuelas de nivel secundario y hospitales desde el ámbito nacional al provincial, que hicieron recaer en estas jurisdicciones mayores funciones sociales hacia la población.

En el ámbito nacional han quedado algunas responsabilidades sociales como los sistemas de jubilaciones y pensiones, algunos programas sociales y la educación universitaria.

La crisis económica global desatada en toda América Latina en la década de los 80 se caracterizó, desde el punto de vista fiscal, por un agotamiento de las fuentes tradicionales de financiamiento por parte del gobierno central. El endeudamiento externo, la utilización de excedentes económicos tanto del sector agroexportador, como los otrora superávit del sistema de seguridad social, y el impuesto inflacionario, constituyeron importantes fuentes de que dispuso el gobierno central, y que no pueden seguir siendo utilizadas para financiar los gastos públicos.

Desde el punto de vista macroeconómico, actualmente existe consenso sobre la importancia que tiene para el programa económico la necesidad de alcanzar el equilibrio fiscal como condición necesaria para el mantenimiento de los objetivos de estabilización.

A partir del año 1991, y fundamentalmente desde la vigencia del plan de convertibilidad y la fuerte desaceleración observada en el nivel general de precios, sumado a los esfuerzos que está realizando el gobierno nacional en materia de administración fiscal, se verificó un fuerte incremento en los recursos tributarios, fundamentalmente los que se coparticipan a las provincias.

En consecuencia, la magnitud de las transferencias en concepto de coparticipación federal de impuestos resulta cuantitativamente mucho más elevada que en el pasado.

Por otra parte, el gobierno nacional, consecuentemente con su filosofía económica, ha eliminado los impuestos a las exportaciones. Por lo tanto, ha desaparecido una de las principales fuentes de financiamiento que le son propias.

Desde el punto de vista del gasto público, la renegociación de la deuda externa plantea compromisos mensuales significativos por parte del gobierno nacional, que se vuelven más gravosos en aquellos períodos en los cuales disminuyen los ingresos derivados de las privatizaciones.

Por todos estos fenómenos, la discusión sobre la distribución de recursos entre la Nación y las Provincias se ha reavivado fuertemente.

El gobierno central argumenta que las provincias disponen de muchos mayores recursos que en el pasado, derivados del importante incremento en la coparticipación federal, por lo cual éstas estarían en condiciones de hacerse cargo de las funciones transferidas. Además, se plantea que las jurisdicciones provinciales no han hecho el esfuerzo suficiente para ajustar sus cuentas, y que el incremento de recursos de origen nacional se traduce automáticamente en mayores erogaciones.

Desde las provincias, se argumenta que el gobierno central, a través de distintas normas jurídicas, ha afectado reiteradas veces la ley de coparticipación federal. En consecuencia, las transferencias efectuadas son menores que las que la ley de coparticipación dispone. Asimismo, se hace hincapié en las mayores responsabilidades sociales asumidas con la comunidad, que requieren ingentes recursos para satisfacer una demanda creciente.

Esto nos lleva a preguntarnos si el sentido del ajuste de las cuentas públicas es el mismo para el gobierno nacional que para las provincias.

Para reflexionar sobre este tema analizaremos en primer término la magnitud relativa de las provincias en relación con el gobierno nacional, y la distribución de funciones entre ambos niveles de gobierno. Creemos que las responsabilidades de los gobiernos provinciales hacia la población constituyen una base a partir de la cual debe evaluarse y diseñarse el sentido del ajuste de las cuentas públicas.

\section{GRADO DE DESCENTRALIZACION}

Argentina muestra un alto grado de descentralización, que puede medirse a través de distintos indicadores:

El gasto provincial representa más del $40 \%$ del gasto total del sector público.

Si excluímos del gasto los intereses internos y externos, la participación de las provincias llega en los últimos dos años al $45 \%$ del gasto total.

El empleo público provincial constituye el 55\% del empleo público total, y el $9 \%$ del empleo global del país.

La tasa de inversión pública de las provincias es de alrededor del $20 \%$ de la tasa de inversión global.

Desde el punto de vista fiscal, las provincias han presentado persistentemente déficit en sus cuentas públicas, y se han caracterizado por una alta dependencia de las transferencias por parte 
del gobierno nacional, ya sea en concepto de recursos coparticipados o aportes.

\section{REASIGNACION DE LAS FUNCIONES ESTATALES ENTRE LA NACION Y LAS PROVINCIAS}

El proceso de descentralización no sólo implicó un mayor grado de concentración del gasto global del sector público en la órbita provincial, sino también un cambio en la asignación de funciones entre la Nación y las Provincias.

Así, el proceso aludido dio lugar a la existencia de "funciones predominantemente nacionales" es decir aquellas en que la mayor responsabilidad por la prestación del servicio es del gobierno central, y "funciones predominantemente provinciales" cuya mayor responsabilidad de prestación se encuentra en las provincias.

Para analizar la distribución de funciones entre los dos niveles jurisdiccionales, las agruparemos en tres categorías.

Por una parte, aquellas vinculadas a la provisión de bienes públicos puros, asociadas a la existencia de un Estado "Gendarme", necesario para la convivencia de las comunidades: defensa, seguridad, justicia, administración de gobierno. Son las denominadas funciones "clásicas", cuyo objetivo es garantizar el orden social necesario para el funcionamiento de los mercados y el desarrollo del sector privado, y que son características de un estado prescindente en materia económica.

Por otra parte, las funciones sociales, que cumplen el rol de garantizar la redistribución de ingresos, características del Estado "Benefactor". Estas funciones se desarrollaron en nuestro país en una etapa de expansión del mercado interno y sustitución de importaciones con tecnologías mano de obra intensivas, y estaban dirigidas a obtener consenso social y legitimidad política. Están integradas por la salud, la educación, la ciencia y técnica, la vivienda, la promoción social, la seguridad social.

En tercer lugar, las funciones correspondientes al "estado productor y regulador", que constituyen un instrumento para la producción y acumulación de capital. Estas funciones se desarrollaron asimismo a partir del período de sustitución de importaciones, como una reacción al proceso de cierre de los mercados internacionales. Se corresponden con políticas intervencionistas en las que el Estado es el vínculo activo de acumulación capitalista. El Estado es uno de los actores principales, no sólo como regulador de la producción y por tanto reasignador de recursos entre sectores sociales, sino como productor de bienes y servicios (1). Estas funciones se refieren a la finalidad "Desarrollo de la Economía" que "abarca la producción de bienes y servicios significativos para el desarrollo económico, incluyendo el fomento, la regulación y el control de la producción del sector privado y de los organismos estatales" (2), y comprenden la actividad de la administración nacional y de las empresas públicas.

A partir de la serie histórica existente, hemos agrupado las diferentes funciones con el criterio expuesto con el objeto de visualizar la participación de las jurisdicciones provinciales en el gasto de la finalidad correspondiente, para todo el sector público consolidado. Esta es una forma de medir el "grado de descentralización” existente para cada grupo de gastos que realiza el sector público con una determinada finalidad.

A estos efectos, consideraremos dos categorías: por un lado, las provincias, y por el otro lado, el "gobierno nacional", que incluye la Administración Nacional, las Empresas del Estado y Entes Binacionales y el Sistema de Seguridad Social.

Hemos extraído de las distintas finalidades aquellas funciones en las cuales las provincias tienen una alta participación.

\section{Cuadro 1}

Grado de Descentralización. Gastos provinciales en \% del total de cada finalidad o función.

\begin{tabular}{lrrrr} 
& $\mathbf{1 9 7 0}$ & $\mathbf{1 9 8 0}$ & $\mathbf{1 9 8 6 / 8 8}$ & $\mathbf{1 9 9 2}$ \\
\hline ESTADO "BENEFACTOR" & 25.9 & 33.8 & 43.1 & 45.4 \\
Otros Gastos sociales & 46.0 & 61.5 & 69.6 & 64.5 \\
Educación & 41.1 & 55.3 & 65.1 & 76.4 \\
Ed. elemental & 62.3 & 94.3 & 96.0 & \\
Ed. media y técnica & 22.4 & 21.2 & 33.1 & \\
Ed. sup. y univers. & 7.3 & 4.5 & 3.0 & \\
Salud & 51.2 & 81.1 & 83.8 & 87.5 \\
Atención médica & 68.5 & 77.1 & 68.1 & \\
Saneamiento ambiental & 28.2 & 39.1 & 61.6 & \\
Vivienda & 52.6 & 94.6 & 94.0 & 93.0 \\
Seguridad Social & 2.1 & 16.5 & 20.4 & 21.1 \\
ESTADO "GENDARME" & 40.0 & 41.9 & 47.5 & 49.7 \\
Administración general & 64.7 & 9.5 & 72.6 & \\
Justicia & 58.6 & 57.4 & 68.8 & \\
Ap.gob.prov.y munic & 100.0 & 100.0 & 100.0 & \\
Defensa & 0.0 & 0.0 & 0.0 & 0.0 \\
Seguridad & 58.0 & 57.8 & 64.9 & \\
Policía interior & 72.5 & 73.1 & 76.8 & \\
ESTADO "PRODUCTOR” & 15.1 & 20.7 & 24.9 & 39.1 \\
DEUDA PUBLICA & 4.0 & 2.4 & 3.0 & 5.9 \\
\hline
\end{tabular}

Fuente: Hasta 1986-88,Secretaría de Hacienda. Erogaciones del Sector Público por Finalidad.- Ministerio de Economía, Obras y Servicios Públicos/PRONATASS. El Gasto Público Social. Buenos Aires, Argentina, 1990. Para 1992, Proyecto de Presupuesto de la Administración Nacional para 1993.

Desde el punto de vista del Estado "Gendarme", podemos identificar algunas "funciones predominantemente provinciales" cuya competencia se ha mantenido en estas jurisdicciones en una alta proporción durante todo el período. Ellas son Justicia, Apoyo a Gobiernos Provinciales y Municipales, Policía Interior.

Las funciones correspondientes al Estado "Productor" son "predominantemente nacionales" durante todo el período.

En consecuencia, en el caso de las funciones citadas, el aná-

(1) En un trabajo anterior, Gasto Público, Rol del Estado y Regímenes Políticos. Argentina 1960-1986 (1988), hemos analizado el surgimiento de estas políticas en los diferentes regímenes políticos y su expresión fiscal.

(2) Ministerio de Economía. Secretaría de Hacienda. Dirección Nacional de Programación Presupuestaria. Normas para la confección del Presupuesto de la Administración Nacional. Ejercicio 1987. 
lisis estático arroja resultados similares al análisis dinámico.

Las consideraciones vinculadas con el Estado "Benefactor" son diferentes. En la función Seguridad Social, la mayor parte de la población está incorporada a un Sistema Nacional de Seguridad Social; existen asimismo algunas cajas nacionales especiales. Una proporción mucho menor corresponde a las cajas provinciales. En consecuencia, el grueso de la competencia es de nivel nacional.

Para el resto de las funciones sociales, las provincias fueron asumiendo a lo largo del tiempo una proporción significativa de funciones sociales. Ello ocurrió fundamentalmente con la educación elemental, la vivienda, la atención médica, el saneamiento ambiental. En este caso, el análisis estático arroja conclusiones diferentes al análisis dinámico, pues este último nos muestra cómo estas funciones se convierten, a lo largo del tiempo, en "funciones predominantemente provinciales".

Las "funciones predominantemente nacionales" son, dentro de las funciones sociales, la Educación Superior y Universitaria, y la Seguridad Social, por las características apuntadas.

Durante el año 1992 se instrumentó el traspaso a las provincias de servicios de educación, salud y minoridad. La mayor transferencia se produce en educación, particularmente de escuelas medias y técnicas, y algunos programas sociales, y representa el $0.6 \%$ del PBI. En consecuencia, la función Educación Media y Técnica pasa a ser una "función predominantemente provincial" ya que aproximadamente el $75 \%$ de la responsabilidad en la prestación de este servicio va a estar en estas jurisdicciones (3).

La finalidad "Deuda Pública" la hemos considerado en forma separada por sus características peculiares. Esta es una función "predominantemente nacional".

Veamos entonces cómo queda configurado el mapa de distribución del gasto en las distintas finalidades, para las provincias y para la Nación.

\section{Cuadro 2}

Distribución de las erogaciones totales por grupos de finalidades (año 1992)

\begin{tabular}{|c|c|c|c|c|}
\hline & \multicolumn{2}{|c|}{ Provincias } & \multicolumn{2}{|c|}{ Gobierno Nacional } \\
\hline & $\%$ PBI & $\%$ & $\%$ PBI & $\%$ \\
\hline Total gasto & 17 & 100 & 23 & 100 \\
\hline Estado "Gendarme" & 21 & 3.5 & 15 & \\
\hline Estado "Benefactor" & 11.5 & 68 & 13.8 & 60 \\
\hline Otros gastos sociales & 9.1 & 54 & 5 & 22 \\
\hline Seguridad Social & 2.4 & 14 & 8.8 & 38 \\
\hline Estado "Productor" & 1.8 & 10 & 2.9 & 13 \\
\hline Deuda Pública & 0.2 & 1 & 2.7 & 12 \\
\hline
\end{tabular}

Fuente: Estimación propia, en base a datos de la Secretaría de Hacienda.

Como puede observarse, en el mapa de asignación de fun- ciones, las provincias tienen un importante Estado "Gendarme", y han ido asumiendo crecientes funciones del Estado "Benefactor", de forma que cerca del $70 \%$ de sus erogaciones constituyen gasto social. La Seguridad Social es una menos significativa parte del gasto. Dentro del Estado "Gendarme" la mayor parte del gasto corresponde a Administración General, y solo una pequeña parte a Seguridad.

Dentro del gobierno nacional, el Estado Benefactor representa el $60 \%$ del gasto total, en el que la mayor proporción corresponde a la Seguridad Social, que alcanza más de un tercio del total. Las funciones asociadas al Estado "Productor y Regulador", en los últimos años se redujeron sustancialmente, ya que en 1989 representaban una cuarta parte del gasto total. Las funciones asociadas al Estado "Gendarme" tienen una magnitud de alrededor del $15 \%$ del total. La deuda pública representa un $12 \%$ del total del gasto. Tanto estos gastos como los de seguridad social incorporan en la programación presupuestaria un importante elemento de rigidez.

Esta diferente distribución y composición del gasto va a tener importantes implicancias dentro de los esquemas con los cuales es factible analizar el ajuste de las cuentas públicas provinciales. En efecto, cualquier diseño de políticas de distribución de recursos entre ambos niveles de gobierno debe tener relación directa con esta estructura funcional del gasto.

\section{AJUSTE NACIONAL Y PROVINCIAL}

Analizaremos si el ajuste de la Nación tiene el mismo significado que el ajuste de las provincias. La Nación acusa a las provincias de realizar pocos esfuerzos para ordenar sus cuentas públicas. Las provincias plantean que su estructura de gastos incorpora requerimientos diferentes a los de la Nación.

Desde el punto de vista macroeconómico, la política presupuestaria se cuenta entre los medios para mantener ciertos objetivos que no se derivan necesariamente de la vigencia del sistema de mercado. Esto se conoce como la función de estabilización de la política presupuestaria. La política presupuestaria constituye un medio para mantener el alto empleo, la estabilidad del nivel de precios y una tasa adecuada de crecimiento económico.

Para el gobierno nacional, responsable de la política macroeconómica, el ajuste fiscal se convirtió en un objetivo prioritario, como condición necesaria para conseguir la estabilidad de precios, y como forma de cumplir con la restricción impuesta por la existencia de fuertes compromisos externos.

Se han conseguido importantes logros en materia de estabilidad de precios. Los acuerdos logrados con los organismos internacionales para el pago de la deuda externa, constituyen un elemento muy importante en relación con la inserción de nuestro país en el contexto mundial. Sin embargo plantea restricciones a la canalización de recursos por parte del gobierno nacional. Si a ello agregamos la alta participación de los gastos de seguridad social, la rigidez es aún mayor.

El ajuste fiscal permanece entonces como un objetivo prioritario en las metas macroeconómicas. Ello tiene varias implicancias, en términos de otros objetivos macroeconómicos. Por una parte, se ve limitada la acción del Estado como promotor

(3) La estimación se realizó en base a los datos contenidos para la función en el Presupuesto de la Administración Nacional 1992 , y la participación provincial del año 1988. 
directo del crecimiento, por el impacto de estas acciones en el gasto público, y asimismo ante el temor de caer en errores cometidos en el pasado, cuando los subsidios contribuyeron a hacer menos competitivos a los sectores económicos, en razón de su utilización indiscriminada.

Por otra parte, el gobierno nacional ha redefinido el rol del Estado, abandonando la producción directa de bienes y servicios, a partir del traspaso a la actividad privada de empresas y sociedades del estado. Asimismo, se ha reducido la actividad reguladora, por lo cual se disolvieron organismos centralizados. Este proceso provocó la disminución del empleo en la administración pública centralizada.

En consecuencia, el objetivo de la estabilidad y el consecuente ajuste fiscal ha implicado que la consecución de otros objetivos, como el empleo, quede en manos de los mecanismos de mercado que corresponden al sector privado de la economía.

La dinámica de la distribución de funciones del gasto público es una consecuencia de este proceso. Un menor estado productor (aunque aún importante), un mayor Estado Gendarme, y el traspaso a las provincias de funciones sociales. La "responsabilidad social" del gobierno nacional está en su mayor parte concentrada en los gastos de seguridad social, y dada la rigidez de estos gastos, se distraen recursos de otras finalidades que el Estado debiera asumir, como los programas destinados a sectores marginados.

Por lo tanto, las necesidades de mantener la estabilidad alcanzada, y el cumplimiento de las metas pactadas con los organismos internacionales, hacen que el gobierno nacional defina una jerarquización de objetivos que tiene el ajuste fiscal como el más importante. En consecuencia, estas mismas restricciones llevan a presionar a las provincias para que prioricen los mismos objetivos: disminuir gastos y empleo, y aumentar recursos, es decir reducir los deficit.

\section{¿Qué ocurre en las provincias?}

Hemos visto que más de las dos terceras partes de los gastos provinciales se corresponde hoy con lo que hemos dado en llamar el Estado benefactor, es decir el gasto social.

La transferencia de servicios sociales a las provincias ha alterado, por otra parte, la composición del gasto social. Cerca del $40 \%$ del gasto del gobierno nacional son las erogaciones en seguridad social. Las provincias tienen una alta concentración de los demás gastos sociales. Esto incorpora una distinción importante. Mientras el gobierno nacional concentra gastos en los que el Estado actúa como agente de transferencia de ingresos, las provincias concentran gastos destinados a la provisión de servicios sociales, como salud y educación.

En consecuencia, esta división de tareas desplaza hacia la órbita provincial aquellas funciones cuya gestión es más complicada, ya que requiere acciones vinculadas con la calidad de las prestaciones, la eficiencia, la gestión de recursos humanos y materiales. Asimismo, la "responsabilidad" social del Estado hacia la población está concentrada mayoritariamente en las provincias. La acción de transferir ingresos que asume el gobierno nacional es de menor complejidad y demanda principalmente ra- cionalidad administrativa.

Esta división de tareas provoca, entre otras cuestiones, que la gestión del gasto tenga en las provincias un carácter más complejo, y que los cambios requieran procesos más lentos que en la Nación.

Asimismo, esta idea se ve reforzada por la existencia de ciertas características inherentes al gasto público social, que impactan sobre el gasto (4).

En primer lugar, se caracteriza por proveer un conjunto de servicios que utilizan intensivamente recursos humanos. Además, esta mano de obra tiene la particularidad de poseer una calificación relativa mayor que en otros sectores del estado. Ejemplo de ello son los maestros, los médicos, las enfermeras. Una primera consecuencia es que el nivel de remuneraciones del sector público afecta una porción sustancial del gasto social.

La transferencia de algunos servicios hacia las provincias, como la educación media, provoca que, ante el mayor nivel de remuneraciones en las provincias que en la Nación, haya una presión hacia arriba de los salarios, y en consecuencia, hacia el alza del gasto social.

Una segunda consecuencia es que la intensidad de recursos humanos del gasto social implica un alto grado de rigidez en el planeamiento y la presupuestación de dicho gasto. Por tanto, los intentos de reducir el gasto total redundan en una baja de los insumos complementarios y bienes de capital, con lo cual disminuye la calidad de los servicios.

La transferencia de servicios a las provincias, con la presión apuntada sobre el nivel de remuneraciones que genera, hace que el mantenimiento de la calidad de los servicios requiera un incremento del gasto total.

En segundo lugar, una de las características que se atribuyen a los bienes y servicios sociales es su alta elasticidad-ingreso de la demanda, es decir son bienes cuya participación en el gasto total de las familias tiende a aumentar a medida que aumenta el ingreso. En consecuencia, cuando hay crecimiento económico, las familias demandan mayor cantidad de servicios sociales. Una parte importante se canaliza hacia el Estado, fundamentalmente en los sectores de ingresos más reducidos.(5) Como en la producción de estos servicios los factores que intervienen no aumentan significativamente su productividad, la mayor demanda requiere un mayor nivel de gasto para su satisfacción.

Aparentemente, la elasticidad-ingreso de los bienes públicos puros y los bienes correspondientes al Estado productor es más baja que la de los bienes sociales; pero aunque no lo fuera, éstos tienen mayor posibilidad de incrementar la productividad per cápita, a través de la introducción de adelanto técnológico o mejora en los procedimientos, con lo cual el aumento de la demanda no debería necesariamente traducirse en un mayor gasto.

En tercer lugar, los bienes y servicios sociales poseen una estructura tecnológica que lleva inevitablemente al incremento progresivo acumulativo de los costos reales en los que hay que incurrir para ofrecerlos. Esto se conoce como hipótesis de Baumol. La razón de ello es que se trata de actividades en las cuales el insumo básico, que es el trabajo, no puede distinguirse del producto final (ejemplos son el dictado de una clase, una

(4) Ver Ministerio de Economía, Obras y Servicios Públicos/PRONATASS. El Gasto Público Social. Buenos Aires, Argentina, 1990.

(5) Esta tendencia se da con mayor intensidad en Educación. En Salud hay una canalización de los sectores de mayores ingresos hacia la oferta privada, aunque los sectores empobrecidos se vuelcan hacia la oferta pública. 
consulta médica). En consecuencia, los aumentos de productividad se verifican de forma muy esporádica. Por otra parte, son actividades para las cuales es muy difícil medir la productividad.

En muchos bienes públicos puros, como así también en aquellos vinculados con el estado regulador y productor, es más sencillo distinguir entre el insumo básico y el producto final, lo cual permite la introducción de tecnologías que aumentan su productividad. En consecuencia, la presión sobre los costos es de menor magnitud que para el gasto social.

Las características apuntadas sobre los bienes y servicios sociales hacen pensar que los procesos de ajuste provincial probablemente sean más lentos y dificultosos que el ajuste nacional. La definición de prioridades en ambos niveles es diferente, en razón de que los objetivos de política son distintos, la vinculación con la población beneficiada es más directa, y debido también a las diferentes estructuras de sus gastos.

El análisis de las cuentas públicas muestra que las provincias han ajustado sus cuentas públicas con menor intensidad que la Nación. El empleo ha crecido en forma significativa, incluso en períodos en los cuales no se verificaron cambios en la asignación de funciones.

Sin embargo, debería analizarse la distribución del empleo en los distintos sectores de gobierno. Por ejemplo, en la provincia de San Juan (6) mientras el empleo en Salud, Educación y Seguridad creció un $25 \%$ en la última década, el empleo en Administración General lo hizo en un $144 \%$. Este comportamiento muestra que los mayores bolsones de ineficiencia se encuentran en los aparatos públicos no asociados con los servicios fundamentales como el gasto social ni con la seguridad de la población.

El gasto social, por su parte, arrastra ineficiencias que han sido identificadas desde su etapa de prestación a nivel nacional. En el sector salud se identifican ciertos problemas, como sobreprestaciones, baja calidad de los servicios, recursos humanos con menor dedicación horaria por los bajos salarios que autogenera exceso de personal. En la educación, relación elevada docente/alumno, alto porcentaje de personal en uso de licencias. Estos elementos deben aún constituir objeto de revisión.

Otra restricción importante que manifiestan las provincias es en cuanto a la calidad de los recursos humanos. Se ha verificado una gran descapitalización humana en gran parte de las jurisdic- ciones. Ello requiere un gran esfuerzo de capacitación, fundamentalmente en los sectores sociales.

Asimismo, existe una gran debilidad de los aparatos de coordinación entre diferentes unidades de gestión provinciales, que se expresa en ineficiencia, ineficacia, falta de información. Ello hace más difícil la programación y la toma de decisiones.

Este conjunto de restricciones hacen que el ajuste provincial tenga características que le son propias, y que se vinculan con el carácter particular de su estructura de gasto, de la calidad de los servicios que presta, y con la conformación de sus recursos humanos.

Por tanto, consideramos que la gestión del gasto en las provincias tiene rasgos diferentes que en la Nación.

\section{REFLEXIONES FINALES}

Entender el ajuste a nivel del gobierno nacional de una forma diferenciada del ajuste a nivel de las provincias tiene importantes consecuencias en términos del replanteo de la distribución de recursos entre los diferentes niveles de gobierno.

Uno de los elementos fundamentales que debe tomarse en cuenta para el replanteo de la distribución de recursos es la diferente estructura del gasto a nivel nacional y provincial, lo cual requiere concebir el ajuste provincial como un proceso más lento y complejo.

El gasto social, que constituye más de la mitad de los gastos provinciales, y consiste fundamentalmente en la provisión de servicios cuya gestión es dificultosa, incorpora un elemento fundamental que no puede dejar de tomarse en cuenta.

En consecuencia, el diseño de políticas de descentralización y su correlato en la transferencia de recursos que requiere, debe partir de la consideración de las funciones que desempeñan los distintos niveles de gobierno hacia la población. Esta cuestión incorpora algunos elementos al debate que no deben soslayarse, como los tiempos en los cuales se ejecutan las políticas, la vinculación entre los recursos y los servicios prestados, y la calidad de estos servicios que el Estado ofrece a la población.

\section{RESUMEN}

A partir del análisis de los procesos de descentralización en la República Argentina, y la configuración actual de la distribución de funciones entre distintos niveles de gobierno, la autora reflexiona sobre el significado del ajuste fiscal a nivel del gobierno nacional y el provincial. En razón de que la mayor parte de los servicios sociales son provistos por los gobiernos locales, y de la característica específica que tienen estos gastos, se concluye que los procesos de ajuste provincial son más lentos y complejos que los del gobierno nacional.

\section{ABSTRACT}

Starting from the analysis of decentralization process in Argentine Republic, and the present configuration of functions distribution between different levels of government, the author make several thougts about the meaning of fiscal adjustment for national government and provinces. Because of the fact that most of the social services are provided by local governments and due to the special characteristic of these expenditures, the conclusion is that adjustment process in provinces are slower and more complex than in national government. 